\title{
Viewership footprint for a low-resource, student- centred collaborative video platform to teach Orthopaedics in Southern Africa
}

\section{Gideon Stephanus Wever ( $\square$ stefanwever@gmail.com )}

Orthopaedic Research Unit, Groote Schuur Hospital, University of Cape Town https://orcid.org/00000001-7072-1565

\section{lan Elliott}

Visiting Orthopaedic Trainee at Department of Orthopaedic Surgery, Groote Schuur Hospital, University of Cape Town South Africa from University of Utah, Salt Lake City, USA

\section{Jeannie McCaul}

Orthopaedic Research Unit, Groote Schuur Hospital, University of Cape Town

\section{Maritz Laubscher}

Orthopaedic Research Unit, Groote Schuur Hospital, University of Cape Town

\section{Robert N Dunn}

Orthopaedic Research Unit, Groote Schuur Hospital, University of Cape Town

\section{Michael Held}

Orthopaedic Research Unit, Groote Schuur Hospital, University of Cape Town

\section{Research article}

Keywords: Training, Clinical skills, YouTube, Education, Videos, Orthopaedics

Posted Date: July 2nd, 2019

DOI: https://doi.org/10.21203/rs.2.10862/v1

License: (a) (1) This work is licensed under a Creative Commons Attribution 4.0 International License. Read Full License 


\section{Abstract}

Introduction Institutions are increasingly using technology to augment the class learning experience of medical students. Especially in Africa, local content is key to allow insights and knowledge to emerge and build transformative capacity for students and patients. There is currently no peer-reviewed video content produced to provide insight into Orthopaedic conditions for medical students and patients in this region. Our goal was to evaluate the demographic and geographic viewership as well as video-specific statistics of orthopaedic teaching videos for medical students on a YouTube channel, with the expressed aim of informing future content production. Methods Videos were produced by local medical students for their in problem-based collaborative projects. Student-owned smartphones and various free video editing software were used to produce these videos, which were then assessed by a group of orthopaedic specialists and uploaded onto a Youtube channel (UCTeach). The analytic reports of this channel generated by Google and YouTube were analysed regarding watch time per day (minutes), average view duration (minutes), most watched videos, top geographies, age, and gender. Results A total of 83 videos of the UCTeachOrtho Channel where uploaded in a two year period with a total watch time of 857062 minutes and 337983 views. The majority of viewers where between the ages 18 and 34 years (85\%). India had the most views $(n=69,089)$ followed by the United States $(n=66,257)$ and South Africa $(n=21,882)$. Most of the videos where watched on mobile phones $(n=183,299)$ and computers $(n=128,228)$. The most watched video was produced in April 2016 on physiological and pathological gait with 51,314 views. Conclusion Our study highlights that a low-cost collaborative video project can lead to high view counts and watch time on YouTube, and is accessible to an audience in low and middle income countries. Student-centred local content in orthopaedic surgery also reached a global audience consistently over a two-year period.

\section{Background}

The use of technology has disrupted the historical pedagogical approach in classrooms and continuously creates new forms of teaching practice. Over the last several years orthopaedic learning has expanded to include online video of topics ranging from surgical techniques to physical exam maneuvers (1). These videos reach a broad audience from orthopaedic faculty to medical students. Student-led creation of media such as educational videos can connect students with external experts, create novel educational content, and stimulate ongoing discussions after formal lessons(2). Open source videos in the classroom, and especially the generation of such videos, can therefore improve participation when used as a learning tool(3). To involve students actively in the content creation of this media creates a student-centred learning process. Here, students become teachers and have greater benefit than students who only learn for their own benefit, also known as the "protégé effect" $(4,5)$. As a result, a studentcentred multimedia learning process can reaches a diverse global audience, and allow the video creators to more deeply understand the content $(6,7)$

These processes could be used in Southern Africa where access to surgical programs is not uniformly available, contributing to one of the lowest surgeon to inhabitant density in the world 0.05-1.3 per 
100,000 (8). Large distances make it cumbersome to connect students to educators, patients to surgeons and surgeons to each other which limits training and educational opportunities, and is aggravated by severe resource restrictions. Multimedia teaching content is therefore an attractive option to reach a broader audience. This is however historically hampered by the cost of production of this teaching content.

The growing availably of internet and smartphone devices in Southern Africa results in increased use of online teaching platforms which allow unique opportunities to overcome some of these challenges(911). Yet, due to significant resource restrictions, there is limited local experience in the role and feasibility of technology and augmented learning for medical students in the form of videos(12).

We aimed to evaluate the viewership statistics of the orthopaedic teaching videos on YouTube created by South African medical students for their peers to understand the demographic and geographic make-up of the viewers and their preferred types of videos. The purpose of understanding these data is to inform targets for future videos and to make improvements to the format of the videos.

\section{Methods}

\section{Videos}

The Orthopaedic Department at the University of Cape Town (UCT) initiated an assessment task for $5^{\text {th }}$ year medical students. Groups of three were given relevant orthopaedic topics, ranging from examination techniques to common orthopaedic problems, with guidelines on how to create an audiovisual presentation no longer than 7 minutes. The videos were graded as part of their final mark of the rotation regarding the content, graphics, technical production, ability to work in a team, and delivery. . The videos were reviewed by orthopaedic specialists and once the content was approved, they were uploaded to the "UCTeach Ortho" YouTube channel created in 2016.

Watch time in minutes for each day and average view duration, top ten watched videos, top geographies, gender, and playback devices were analysed.

\section{Statistics}

The standard analytic reports from February 2016 (date of inception) to March 2019 made available by Google and YouTube (as its subsidiary) were downloaded for analysis from the YouTube studio on the channel. Descriptive statistics were used to evaluate the analytics. Means were compared for each category in each subsection. The raw data provided by Google/YouTube didn't allow for the granularity necessary to calculate the confidence interval or standard deviations for most metrics, though for those metrics that were reported in means, mean and $95 \%$ confidence intervals were calculated. 


\section{Results}

A total of 83 videos where uploaded in the two year period with a total watch time of 857062 minutes and 337983 views.

Most viewers were between the 18 and 34 years of age (85\%) with predominantly male viewer between 25 and 35 years of age (30.4\%) (Table 1). Most views were from India ( $n=69089 ; 32.1 \%)$ followed by the United States $(n=66257 ; 30.7 \%)$ and South Africa $(n=21882 ; 10.2 \%)$ (Figure 1). Videos were watched by 892 viewers in low income countries, for a total watch time of 1,792 minutes. The majority of these viewers were from Nepal $(n=602)$, though 8 different low income countries in Asia and Africa were represented (Figure 2). 922 Viewers from 16 different African countries (15 outside of South Africa) watched the videos (Figure 3). Low income countries accounted for $10.1 \%$ of the viewership (Table 2 )

Most of the videos where watched on mobile devices $(n=183299 ; 53.8 \%)$ and computers $(n=128228$; $37.6 \%$ ) (Figure 4). The average watch time per video was 2.16 minutes ( $95 \% \mathrm{Cl} 2.00$ to 2.32 minutes), though the average length of each video was 6.71 minutes ( $95 \% \mathrm{Cl} 6.30$ to 7.12 minutes). The top 10 videos regarding watch time (Figure 5) used predominantly Khan-style tablet drawings and pictures rather than face recording of a person talking. The most watched video was on physiological and pathological gait with a total of 51,314 views (Figure 5) Although the video was 7.48 minutes in duration the average viewing duration was only 2.9 minutes. The most watched video in the United States was "How to do a bier's block" and in India, "Management of tuberculosis of the spine". Videos specifically made for patient education had very low view counts of less than 100 per video.

\section{Discussion}

Our study showed that a low-cost student-centred collaborative production of Orthopaedics teaching videos specific to Southern Africa led to high viewing counts and watch time on an open source platform. Interestingly, the majority of views originated outside of the targeted audience of South Africa.

Most viewers in South Africa were at the age of medical students in the country, between the age of 18 and 24 and younger than viewers from India or the US. A possible reason for this could be that those producing the videos in South Africa discussed the existence of the videos with their peers. The average ages of viewers in the United States fall into the age range typical for resident trainees, as US medical students average age as first years is 23 and 26 by senior year (i.e. when they graduate to enter residency)(13). The average ages of viewers in India does fall into the ages expected of medical students, as the average age of medical students in India is around 20 years old(14). We make an assumption that most viewers are in some state of medical training, though this is not actually knowable based on the data collected. This study does show that videos produced in a middle income country can and do reach audiences in both low and middle income countries at relatively high view rates.

We also found the viewing time per video was much shorter than its duration and decreased from approximately six minutes in 2016 to between two to three minutes in the following years. In a study of 
student-video engagement within e-learning environments, Guo et al found that video duration longer than 6 minutes decreased student engagement with the material, and that this disengagement dropped off preciptiously with only 50\% engagement with videos of between 9-12 minutes long(15). There could be many reasons for the short viewing time of these videos. The time watched could be indicative of attention span, or of the time it took to convey the major point of the video. Either way, the actual watched duration could be used as a guide for future videos.

The most successful videos in terms of viewership used Khan-style tablet drawings, named after the Khan Academy style of educational video which employs sketching out the lesson with audio, similar to drawing on a whiteboard or chalkboard. Khan style drawing focuses on a live drawing rather than video of a teacher's face during explaining (16). Similarly, It has been shown before that short videos, Khanstyle drawings and informal talking-head videos are more engaging than pre-recorded high-quality classroom videos(15). It should be noted that the content was created using basic equipment like smartphone cameras and free video editing software. The production of such videos was therefore not restricted by a lack of resources.

The most popular videos were those which reached a broad training audience, such as those on clinical skills. These videos applied to audiences both in Southern Africa but also places like the United States. The video "Management of TB spine" was viewed 12756 times. Although it is not known from what geographical areas these views originated from the overall viewership was from countries with high burden (17).

Some of the videos which were specifically produced for patient education. These had low view counts and viewing time. The low numbers of views for these videos indicates either that patients fail to search successfully for these videos, or that there is low access to the internet due to the low-income nature of the targeted patients. To increase access, videos could therefore be shown in the hospital and clinic facilities much like patient focused educational programs in the United States targeting arthroplasty patients(18,19).

\section{Limitations}

There are several limitations to this study. This is a retrospective study using analytics from a third party website regarding viewership, therefore data interpretation is entirely based upon the quality of data collection by Google. For the purpose of this study, it is assumed this is done in a rigorous manner. The field of work or study of the viewers is unknown and thus it is unclear what percentage of viewers are actually in the medical field, and if they are, at what stage of training. These videos were produced for a specific audience in Southern Africa, though they obviously have some appeal to a broader audience, and there is little direct feedback on what subjects to explore or how to improve the video presentations.

\section{Conclusions}


Our study has highlighted that a low cost problem-based collaborative video project can lead to high viewing counts and watch time on YouTube in South Africa and reach Indian and American viewers, as well as viewers in low and middle income countries. The average age of our viewers and that of medical students matched, especially in South Africa. Videos using khan-style drawings and informal talkinghead videos had high view counts. The average watch time was less than 3 minutes indicating the target for acceptable video duration for viewers.

The local student-based production of the videos was viable and successful in terms of continuous production of videos, reach and viewership.

\section{Declarations}

\section{Contributions of authors, competing interests and funding}

SW wrote protocol, analysed the data and wrote the final manuscript.

JM contributed to the concept and design of the video project. ML and RD contributed to the design of the project and critically reviewed the final manuscript. IE analysed the data and critically reviewed the final manuscript. MH conceptualized and designed the study, contributed to the analysis and interpretation of data and oversaw the writing of the manuscript. Supporting data can be accessed through contacting the corresponding author.

\section{Conflict of interest}

All authors confirm that no benefits of any form have been received from any party related directly or indirectly to the subject of this article.

All authors hereby declare that they have no competing interests to disclose.

\section{Ethical Clearance}

Ethics clearance was obtained from the Faculty of Health Sciences Human Research Ethics Committee, University of Cape Town. (HREC 185/2018)

\section{References}

1. VuMedi [Internet]. [cited 2019 Mar 15]. Available from: https://www.vumedi.com/accounts/login/

2. George DR, Dellasega C. Use of social media in graduate-level medical humanities education: two pilot studies from Penn State College of Medicine. Med Teach. 2011;33(8):e429-e434. 
3. Buzzetto-More N. Student attitudes towards the integration of YouTube in online, hybrid, and webassisted courses: An examination of the impact of course modality on perception. J Online Learn Teach. 2015;11(1):55.

4. Kristensen P, Bjerkedal T. Explaining the relation between birth order and intelligence. Science. 2007;316(5832):1717-1717.

5. Chase CC, Chin DB, Oppezzo MA, Schwartz DL. Teachable agents and the protégé effect: Increasing the effort towards learning. J Sci Educ Technol. 2009;18(4):334-352.

6. Eick CJ, King Jr DT. Nonscience Majors' Perceptions on the Use of YouTube Video to Support Learning in an Integrated Science Lecture. J Coll Sci Teach. 2012;42(1).

7. Duffy P. Engaging the YouTube Google-eyed generation: Strategies for using Web 2.0 in teaching and learning. Electron J E-Learn. 2008;6(2):119-130.

8. Dell AJ, Gray S, Fraser R, Held M, Dunn R. Orthopaedic Surgeon Density in South Africa. World J Surg. 2018;42(12):3849-55.

9. Poushter J. Smartphone ownership and internet usage continues to climb in emerging economies. Pew Res Cent. 2016;22:1-44.

10. Romanov K, Nevgi A. Do medical students watch video clips in eLearning and do these facilitate learning? Med Teach. 2007 Jan 1;29(5):490-4.

11. Nicole A. An Examination of Undergraduate Student's Perceptions and Predilections of the Use of YouTube in the Teaching and Learning Process. Interdiscip J E-Ski Lifelong Learn. 2014;10(unknown):017-032.

12. Frehywot S, Vovides $\mathrm{Y}$, Talib Z, Mikhail N, Ross $\mathrm{H}$, Wohltjen $\mathrm{H}$, et al. E-learning in medical education in resource constrained low-and middle-income countries. Hum Resour Health. 2013;11(1):4.

13. Frank E, Carrera J, Elon L, Hertzberg V. Basic Demographics, Health Practices, and Health Status of U.S. Medical Students. Am J Prev Med. 2006 Dec;31(6):499-505.

14. Iqbal S, Gupta S, Venkatarao E. Stress, anxiety \& depression among medical undergraduate students \& their socio-demographic correlates. Indian J Med Res. 2015 Mar;141(3):354-7.

15. Guo PJ, Kim J, Rubin R. How video production affects student engagement: An empirical study of MOOC videos. In: Proceedings of the first ACM conference on Learning@ scale conference. ACM; 2014. p. 41-50.

16. Khan Academy [Internet]. Khan Academy. [cited 2019 Mar 15]. Available from: http://www.khanacademy.org

17. Sharma A, Hill A, Kurbatova E, van der Walt M, Kvasnovsky C, Tupasi TE, et al. Estimating the future burden of multidrug-resistant and extensively drug-resistant tuberculosis in India, the Philippines, Russia, and South Africa: a mathematical modelling study. Lancet Infect Dis. 2017 Jul 1;17(7):70715.

18. Yoon RS, Nellans KW, Geller JA, Kim AD, Jacobs MR, Macaulay W. Patient Education Before Hip or Knee Arthroplasty Lowers Length of Stay. J Arthroplasty. 2010 Jun 1;25(4):547-51. 
19. Lin PC, Lin LC, Lin JJ. Comparing the effectiveness of different educational programs for patients with total knee arthroplasty. Orthop Nurs. 1997 Oct;16(5):43-9.

\section{Tables}

\begin{tabular}{lr}
\hline Viewer age & Percentage of Viewers \\
\hline 18-24 years & 37.2 \\
25-34 years & 48.3 \\
\hline $35-44$ years & 13.1 \\
\hline $45-54$ years & 1.4 \\
\hline \hline
\end{tabular}

Table 1: Viewer Age

$\mathrm{n} \%$ of viewing Countries

\begin{tabular}{lcc}
\hline High Income & 34 & 43.0 \\
Middle Income & 37 & 46.8 \\
\hline Low Income & 8 & 10.1 \\
\hline \hline
\end{tabular}

Table 2: Percentage representation in viewership by income status

\section{Figures}

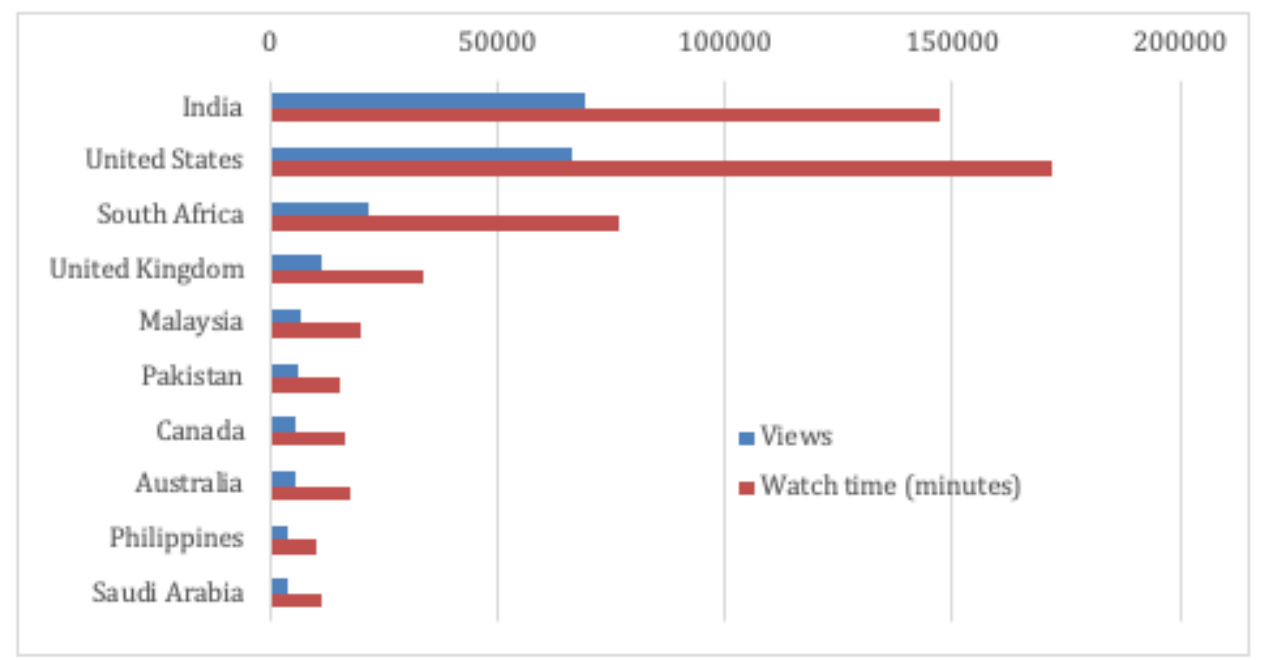

\section{Figure 1}

Countries with Most Views 


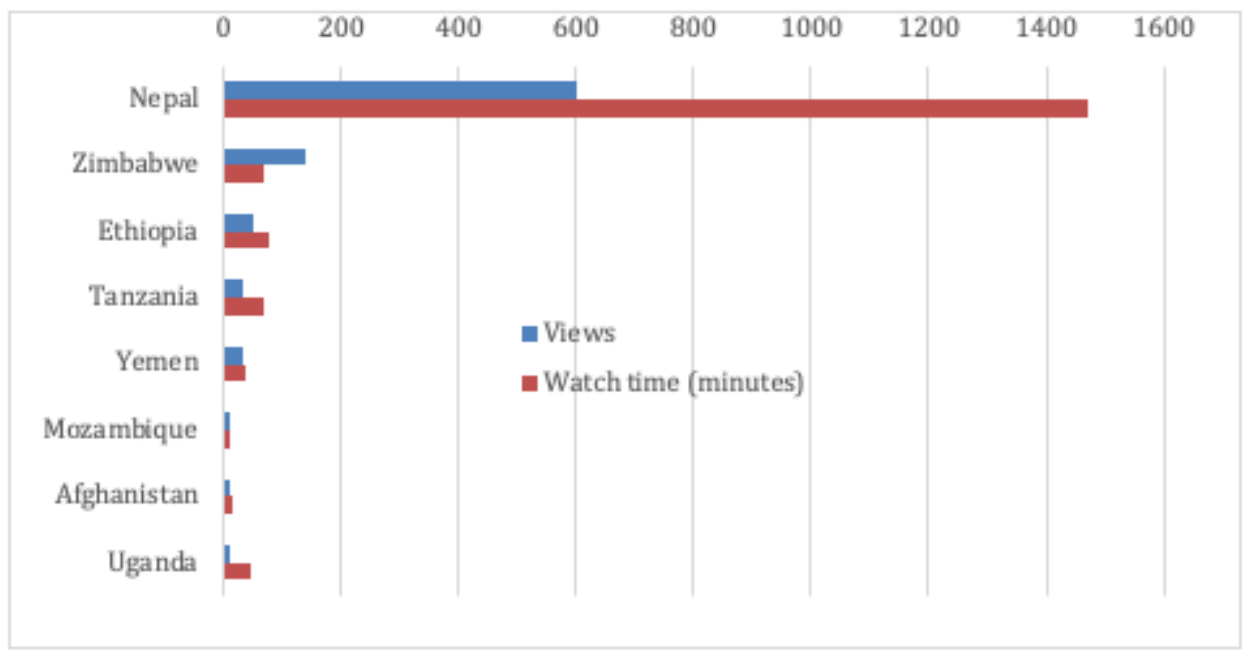

Figure 2

Low Income Countries by Number of Views

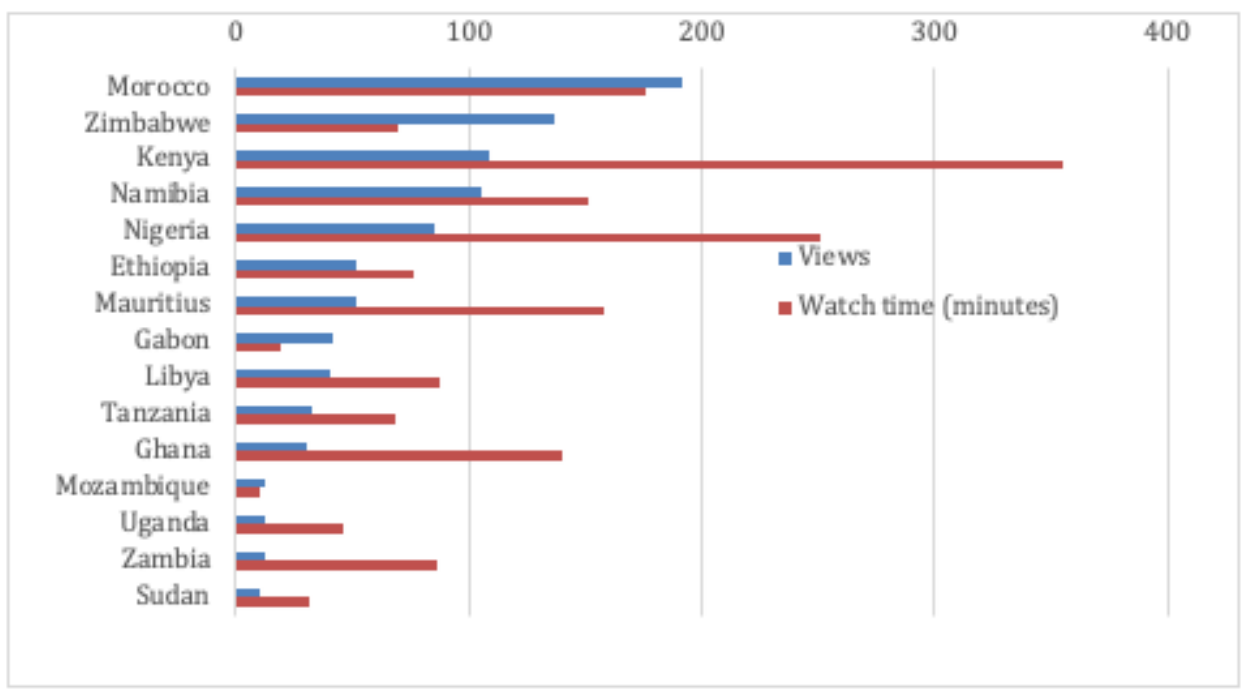

\section{Figure 3}

\section{African Countries by Number of Views}




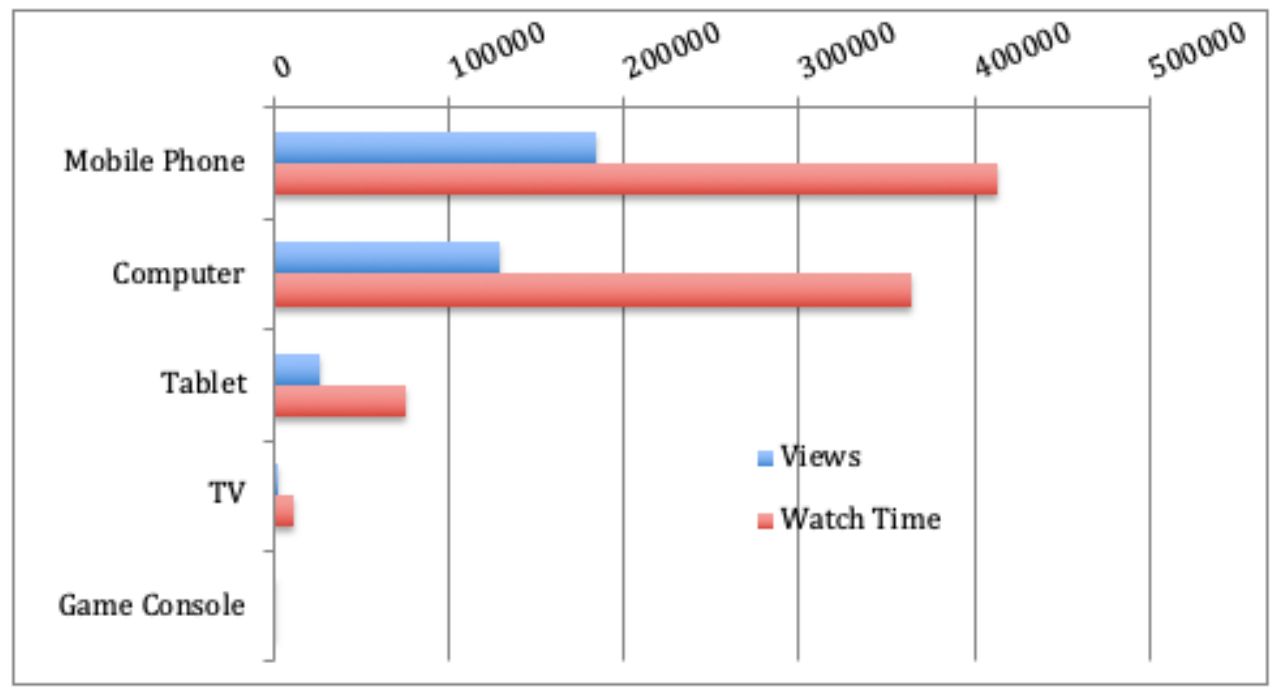

\section{Figure 4}

\section{Device Time by Number of Views}

\begin{tabular}{|c|c|c|c|c|}
\hline & ${ }_{40,000}$ & 80,000 & 120,000 & 260,0 \\
\hline Physiologic and Pathologic gait & 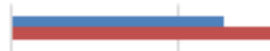 & & & \\
\hline How to do a digital block & . & & & \\
\hline How to look at C-spine X-Rays & & 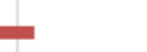 & & \\
\hline Thomas Traction Splint for femur fractures -... & ـ & & & \\
\hline Osteomyelitis & L & - Views & & \\
\hline Approach to Orthopedic Radiography & 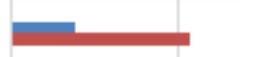 & & & \\
\hline How to do a Bier Block & & & & \\
\hline ASIA Impairment Scale and Spinal Cord Injuries & 工 & & & \\
\hline Management of TB Spine & س & & & \\
\hline Asia Impairment scale & 口 & & & \\
\hline
\end{tabular}

\section{Figure 5}

Number of Views per Video Title 